\title{
Microarray and ChIP-seq data analysis revealed changes in p53-mediated transcriptional regulation in Nutlin-3-treated U2OS cells
}

\author{
SONG ZHAO ${ }^{1}$, FENG NIU ${ }^{1}$, CHANG-YAN XU ${ }^{2}$, LONG YE ${ }^{1}$, GUI-BIN BI ${ }^{1}$, \\ LIN CHEN $^{1}$, PING GONG ${ }^{1}$, GANG TIAN ${ }^{1}$ and TIAN-HONG NIE ${ }^{1}$ \\ Departments of ${ }^{1}$ Spinal Surgery and ${ }^{2}$ Medical Records, The First Hospital \\ of Jilin University, Changchun, Jilin 130021, P.R. China
}

Received September 11, 2014; Accepted May 27, 2015

DOI: $10.3892 / \mathrm{mmr} .2015 .3933$

\begin{abstract}
Integrative analysis of chromatin immunoprecipitation-sequencing (ChIP-seq) data and microarray data was performed to illustrate the effect of Nutlin-3 on promoter selectivity and transcriptional regulation by the tumor suppressor p53 in U2OS human osteosarcoma cells. Raw data (accession number, GSE46642) were downloaded from Gene Expression Omnibus. Differential analyses were performed using package limma of $R$ software. Gene ontology enrichment and Kyoto Encyclopedia of Genes and Genomes pathway enrichment analyses were performed for the differentially expressed genes (DEGs) using the Database for Annotation, Visualization and Integration Discovery. Integrative analysis of ChIP-seq data and microarray data were confirmed with ChIP-Array. A total of 565 DEGs were identified, including 373 upregulated genes and 192 downregulated genes. Genes involved in the p53 signaling pathway, cell cycle, DNA replication, cytokine-cytokine receptor interaction and melanoma were markedly over-represented in the DEGs. A total of 39 DEGs were directly regulated by p53 and two were the transcription factors (TFs), E2F2 and HOXA1. E2F2 regulated 25 DEGs, while HOXA1 regulated one DEG. The cell cycle, p53 signaling pathway, melanoma and pathways involved in cancer were enriched in the direct and indirect target genes. Changes in the p53-binding pattern induced by Nutlin-3 were described in the present study, which may advance the understanding of the regulatory network of p53 in osteosarcoma and aid in the development of novel therapies.
\end{abstract}

Correspondence to: Dr Feng Niu, Department of Spinal Surgery, The First Hospital of Jilin University, 71 Xinmin Street, Changchun, Jilin 130021, P.R. China

E-mail: niufniu@163.com

Key words: p53, osteosarcoma, transcription factor, chromatin immunoprecipitation-sequencing data, differentially expressed genes, functional enrichment analysis

\section{Introduction}

Gene transcription is regulated by dynamic interactions between cis-regulatory elements and regulatory proteins, including transcription factors (TFs). Tumor protein p53 is an important $\mathrm{TF}$ involved in various cellular processes, including growth arrest, senescence and apoptosis (1-3). Following cellular stress, stabilized p53 translocates into the nucleus and subsequently binds to the consensus sequence motif to regulate the expression of hundreds of genes.

p53 is critical in tumor suppression and loss of p53 function is required for cancer progression. Mutational inactivation of p53 is detected in $>50 \%$ of human cancer types (4). A number of downstream proteins of p53 have been identified (5-7). Nevertheless, several of the factors expected to affect p53-induced changes in gene expression are poorly understood, including the impact of different stresses that can induce p53. Genome-wide studies may provide an improved understanding of its transcriptional regulatory functions in certain types of cancer (8-10), including osteosarcoma.

Osteosarcoma is the eighth most common type of childhood cancer and is also the most common histological form of primary bone cancer (11). The mortality rates for osteosarcoma have been declining by $\sim 1.3 \%$ annually (12). The overall 5 -year survival rate for osteosarcoma is $\sim 68 \%$ (12). Future studies are required to fully disclose the molecular mechanisms and advance therapeutic development.

In the present study, human U2OS osteosarcoma cells, expressing wild-type $\mathrm{p} 53$, were used to investigate the effect of treatment with Nutlin-3 (a non-genotoxic activator of p53) on p53 binding genes. Different from a previous study by Menendez et al (13), a stricter threshold [ $\left[\log _{2}\right.$ fold change (FC) $\mid>1$ and false discovery rate (FDR) $<0.05$ vs. FC $>2$ and FDR $\leq 0.1]$ was used to select the differentially expressed genes (DEGs) and to construct the regulatory association between p53 and its target genes.

\section{Materials and methods}

Raw data. The raw data (accession number, GSE46642) were downloaded from Gene Expression Omnibus (http://www. 
ncbi.nlm.nih.gov/geo/), including chromatin immunoprecipitation-sequencing (ChIP-seq) data (accession number, GSE46641; three Nutlin-3 treated U2OS cell samples) and microarray data (accession number, GSE46493; three Nutlin-3 treated U2OS cell samples and three control samples). Gene expression levels were measured using Affymetrix Human Genome U133 Plus 2.0 Array (Affymetrix Inc., Santa Clara, CA, USA).

Pre-treatment and differential analysis. The microarray data were read using the package, affy (14), on the software $R$ (http://www.r-project.org/). Following background correction and normalization with a Robust Multi-array Analysis (RMA) method in $R$ affy, the gene expression levels were determined. Differential analysis was performed using the package, linear models for microarray data (limma) (15), on the software $R$. Multiple-testing correction was performed using the Bayes method (implemented in the 'limma' $R$ package). The following threshold was set for the screening of the DEGs: $\log _{2} \mathrm{FCl}>1$ and $\mathrm{FDR}<0.05$.

Integrative analysis of microarray data and ChIP-seq data. ChIP-Array (http://jjwanglab.org/chip-array) is an online tool developed for integrative analysis of microarray data and ChIP-seq data (16). It identifies the indirect target, Z, by identifying an intermediate transcription factor (TF), Y, which is a putative regulator of $Z$ and a target of $X$. The putative regulator of $\mathrm{Z}$ is identified by scanning all promoters in the genome with position weight matrix (PWMs) of all Ys from three publicly accessible databases [JASPAR (http://jaspar.genereg. net), UniPROBE (http://uniprobe.org) and TRANSFAC (http://www.gene-regulation.com/pub/databases.html) derived transcription factor binding site database from University of California, Santa Cruz genome browser] (16).

In the present study, the parameters were set as follows: Promoter range, -500 +100; TF database, UniPROBE; PWM scan P-value, $10^{-5}$; and conservation filtering P-value, 0.001 . Finally, a gene regulatory network was obtained for $\mathrm{p} 53$, including its direct and indirect target genes.

Functional enrichment analysis. Gene Ontology (GO) and Kyoto Encyclopedia of Genes and Genomes (KEGG) pathway enrichment analyses were performed for the DEGs using the Database for Annotation, Visualization and Integration Discovery (http://david.abcc.ncifcrf.gov/) online tools (17). $\mathrm{P}<0.05$ was considered to indicate a statistically significant difference and was set as the cut-off.

\section{Results}

Differentially expressed genes. Gene expression data prior to and following normalization with the RMA method are demonstrated in Fig. 1. A good performance of normalization was achieved.

A total of 565 DEGs were identified, including 373 upregulated genes and 192 downregulated genes. Clustering and a heat-map of the expression values for DEGs are shown in Fig. 2. The Nutlin-3 treated U2OS samples were well distinguished from the control samples, suggesting the reliability of the DEGs.

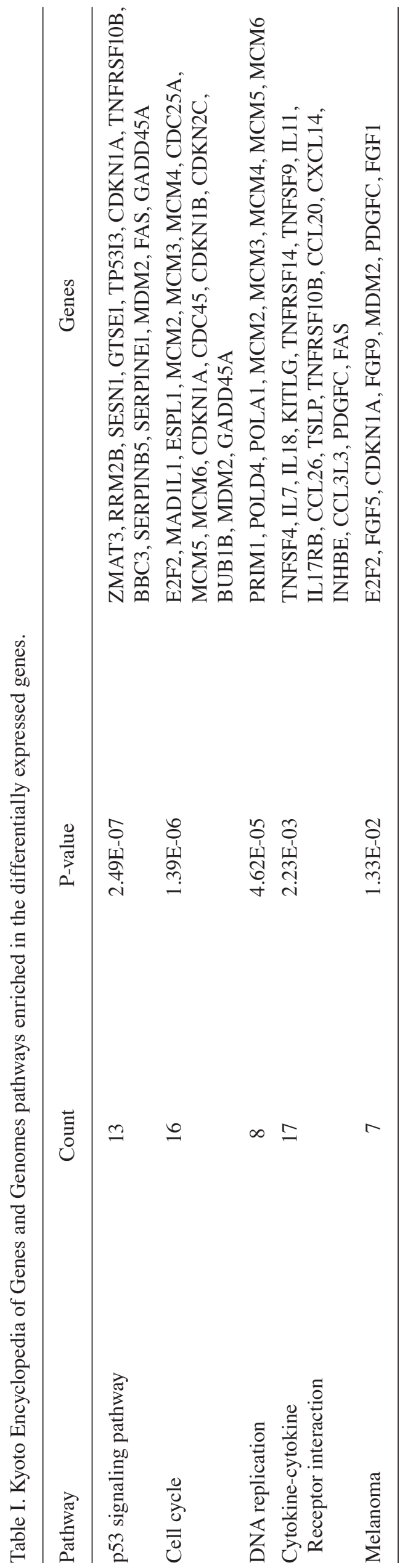


Table II. Kyoto Encyclopedia of Genes and Genomes pathways enriched in the direct and indirect target genes of p53.

\begin{tabular}{lccl}
\hline Pathway & Count & P-value & \multicolumn{1}{c}{ Genes } \\
\hline Cell cycle & 5 & $1.01 \mathrm{E}-03$ & E2F2, CDKN1B, MDM2, \\
& 3 & $2.60 \mathrm{E}-02$ & MCM2, CDC25A \\
p53 signaling pathway & 3 & $2.82 \mathrm{E}-02$ & TNFRSF10B, ZMAT3, MDM2 \\
Melanoma & 5 & $3.04 \mathrm{E}-02$ & E2F2, MDM2, FGF1 \\
Pathways in cancer & 3 & $3.12 \mathrm{E}-02$ & WNT5A, E2F2, CDKN1B, \\
Chronic myeloid leukemia & & & MDM2, FGF1 \\
\hline
\end{tabular}
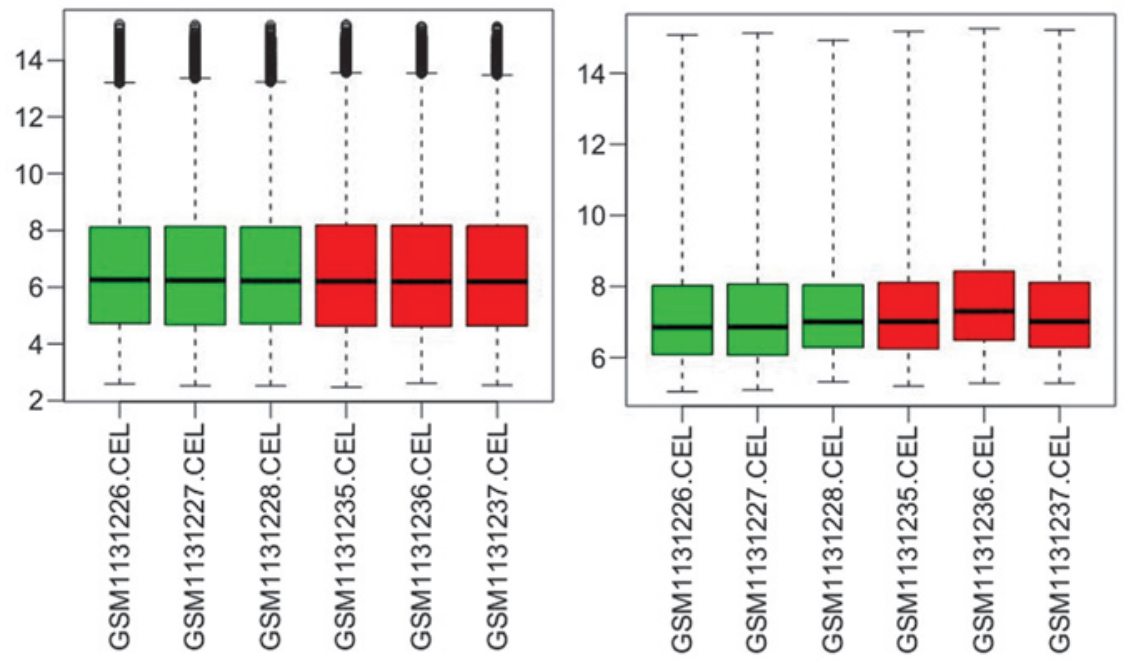

Figure 1. Box plots of gene expression data prior to (left) and following normalization (right). Nutlin-3 treated U2OS samples are shown in red and control samples are in green.

Functional enrichment analysis result. The top 10 GO terms are listed in Fig. 3. Nuclear division, the response to abiotic stimulus, positive regulation of cell proliferation and cell cycle were significantly enriched in the DEGs.

The KEGG pathways with $\mathrm{P}<0.05$ are listed in Table I. The p53 signaling pathway, cell cycle, DNA replication, cytokine-cytokine receptor interaction and melanoma were significantly over-represented in the DEGs.

Transcriptional regulatory network of p53. Integrative analysis of ChIP-seq data and microarray data was performed using the ChIP-Array online tool. A total of 39 DEGs were directly regulated by $\mathrm{p} 53$, and two of them were TFs: E2F transcription factor 2 (E2F2) and homeobox A1 (HOXA1). E2F2 regulated 25 DEGs and HOXA1 regulated one DEG (Fig. 4).

Functional enrichment analysis result of the target genes. GO enrichment analysis was performed for the direct and indirect target genes of p53 (Fig. 5). Cell cycle and cell-cell signaling were included in the list.

The KEGG pathway enriched in all the target genes of p53 were also disclosed (Table II), including cell cycle, p53 signaling pathway, melanoma and pathways in cancer.

\section{Discussion}

In the present study, a total of 565 DEGs were identified in Nutlin-3-treated U2OS cells compared with the control samples. Of these DEGs, 373 were upregulated genes and 192 were downregulated genes. Functional enrichment analysis revealed that the p53 signaling pathway, cell cycle and DNA replication were significantly over-represented in the DEGs. This result suggested the importance of p53 in osteosarcoma. p53 functions as a cell cycle control protein in osteosarcoma (18) and the presence of p53 mutations in human osteosarcoma is correlated with high levels of genomic instability (19), confirming the critical importance of p53 in response to stresses, including DNA damage. Berman et al (20) reported that metastatic osteosarcoma is induced by the inactivation of $\mathrm{Rb}$ and p53 (20). The comparative analysis of gene expression profiles between Nutlin-3-treated U2OS cells and controls further described the critical importance of p53 in osteosarcoma. Notably, p53 gene therapy of human osteosarcoma is also suggested and has been previously investigated (21).

To further illustrate the changes in the p53-binding pattern in response to treatment with Nutlin-3, integrative analysis of microarray data and ChIP-seq data was performed and the transcriptional regulatory network of p53 was obtained. A total of 39 DEGs were directly regulated by p53 and two of which 


\section{Color Key}

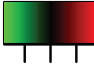

$\begin{array}{ll}-1 & 1\end{array}$
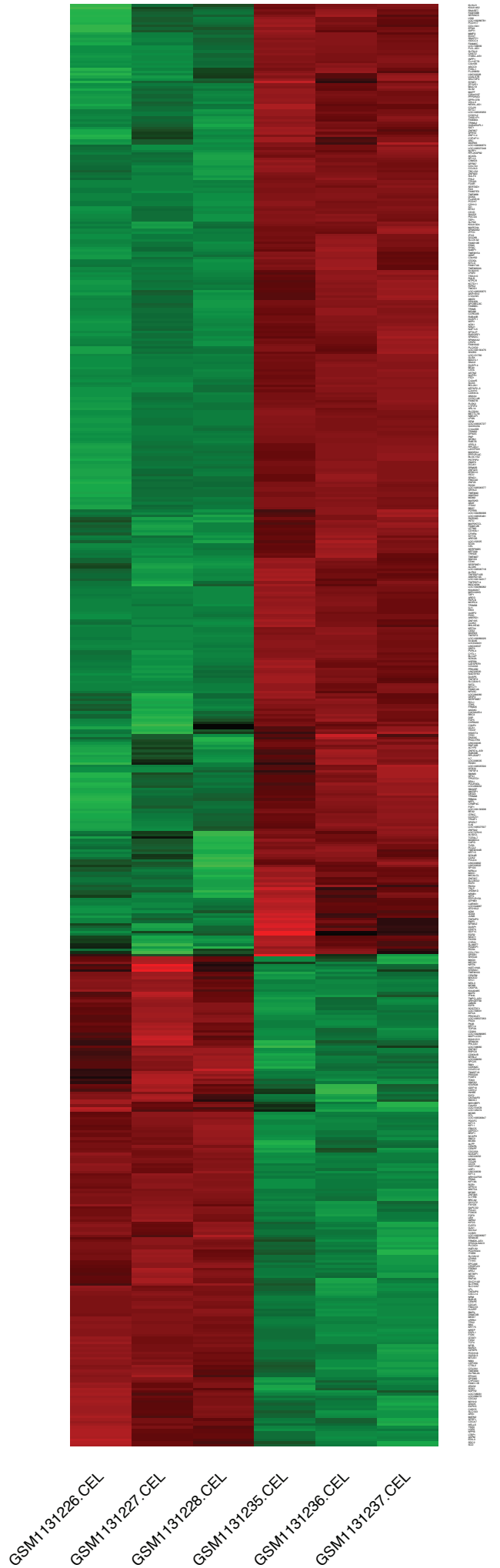

Figure 2. Clustering and heatmap of expression values for differentially expressed genes. Downregulated genes are shown in green and upregulated genes are in red. From left to right, the first three samples are nutlin-3-treated U2OS samples and the latter are three control samples.
A

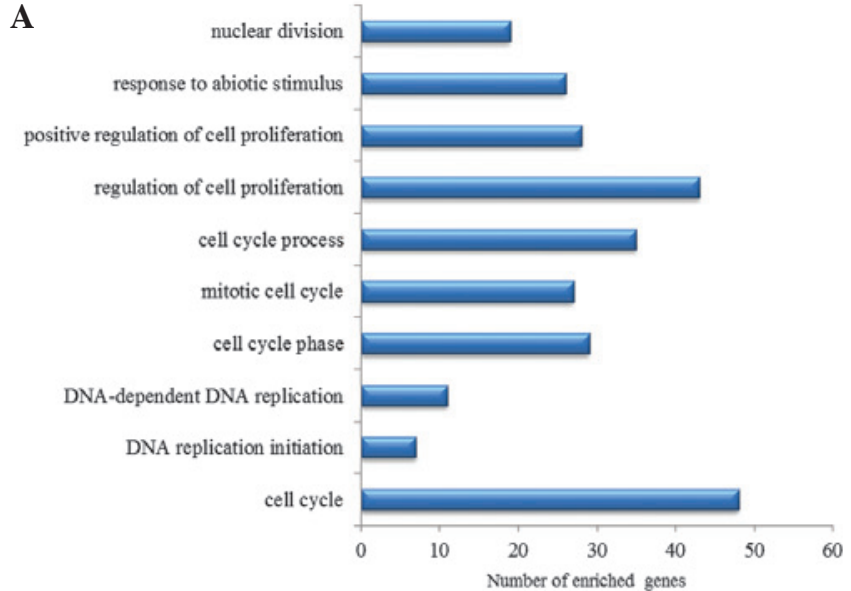

B

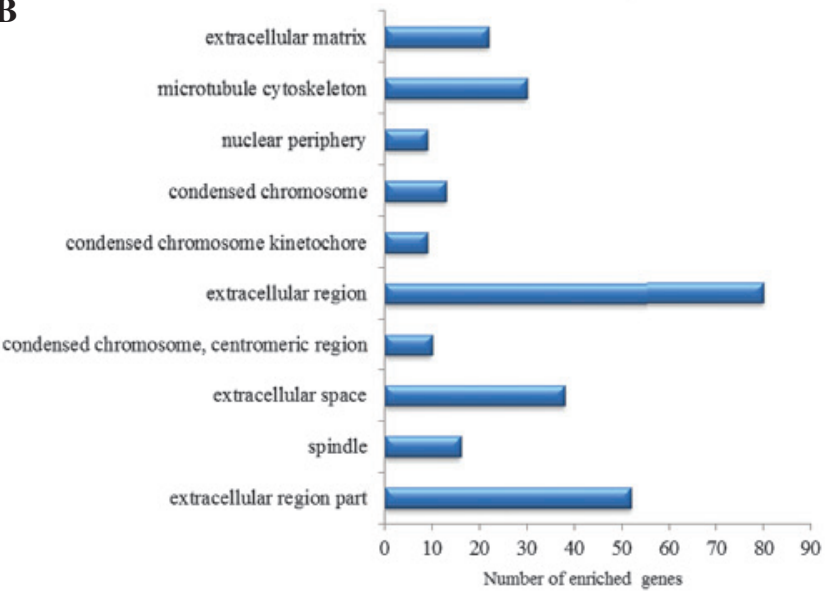

C

protein serine/threonine kinase inhibitor activity

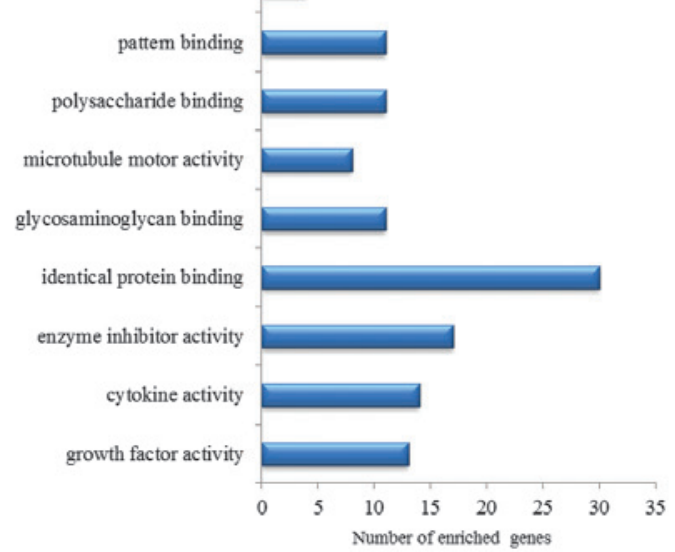

Figure 3. Gene ontology term enriched in the differentially expressed genes. (A) Biological process, (B) cellular component and (C) molecular function.

were the TFs, E2F2 and HOXA1. E2F2 regulated 25 DEGs and HOXA1 regulated only one DEG. Functional enrichment analysis demonstrated that the cell cycle, p53 signaling pathway, melanoma and pathways in cancer were enriched in the direct and indirect target genes, further confirming the critical importance of p53 in osteosarcoma. It may be beneficial to further investigate these target genes to reveal the complete molecular mechanisms and provide potential therapeutic targets.

Several direct target genes of p53 have been confirmed to be involved in tumorigenesis. The MDM2 proto-oncogene 


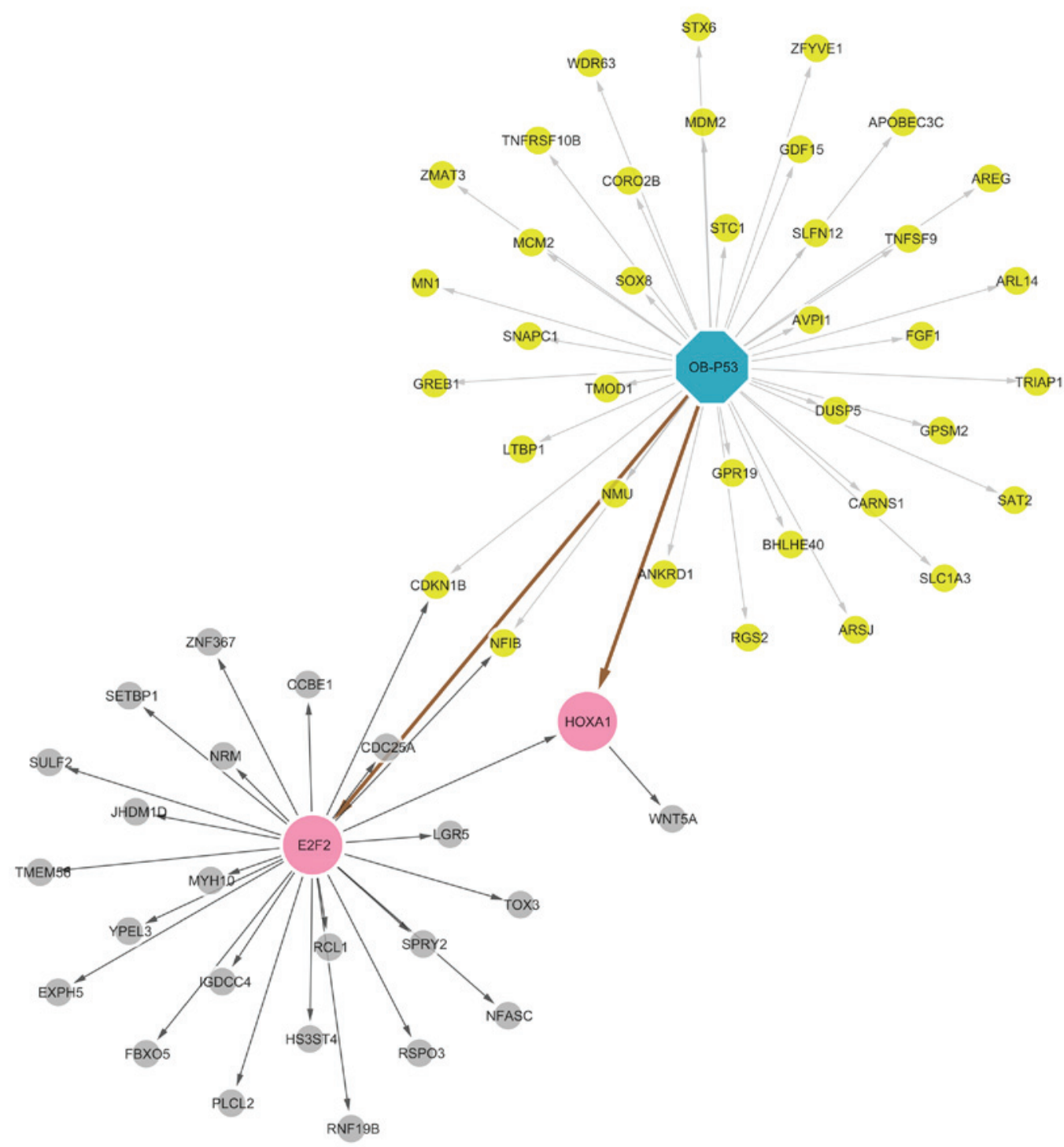

Figure 4. Transcription regulatory network of p53. p53 is shown in blue and its direct target genes are in yellow. Two transcription factors are shown in pink and their target genes are in grey. E2F2, E2F transcription factor 2; HOXA1, homeobox A1.

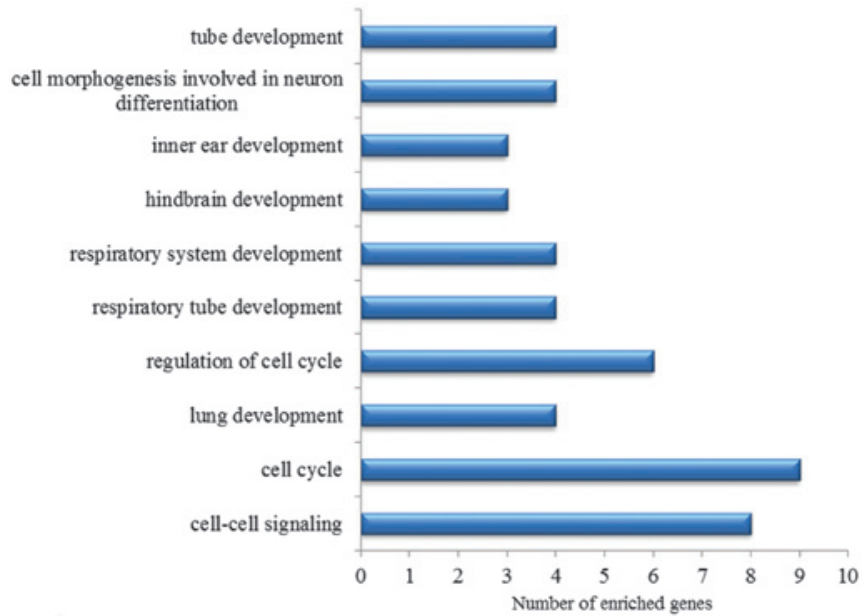

Figure 5. Gene ontology biological process terms enriched in the direct and indirect target genes of p53.

is a nuclear-localized E3 ubiquitin ligase. MDM2 promotes tumor formation by targeting p53 for proteasomal degrada- tion (22). The gene is itself transcriptionally regulated by p53. Therefore, targeting the p53-MDM2 interaction is 
hypothesized as a cancer therapeutic agent $(23,24)$. Syntaxin 6 (STX6) is a regulator of the protein trafficking machinery. Zhang et al (25) indicated that STX6 is an effector and a modulator of the p53 family in the regulation of cell adhesion and survival. Fibroblast growth factor 1 (FGF1) is a member of the FGF family. Bouleau et al (26) indicated that FGF1 inhibits p53-dependent apoptosis and cell cycle arrest via an intracrine pathway. Meningioma 1 stimulates vitamin D receptor-mediated transcription and inhibits osteoblast cell proliferation (27). It is required for appropriate osteoblast proliferation, motility, differentiation and function (28). The present study hypothesized that this protein may be a novel target to modulate osteosarcoma cell growth.

E2F2 and HOXA1 are directly targeted by p53 and they are also TFs. It has been confirmed that E2F2 inhibits tumorigenesis $(29,30)$. E2F activity is critical for the control of the G1 to $\mathrm{S}$ phase transition. Laresgoiti et al (31) demonstrated that E2F2 and $\mathrm{CREB}$ cooperatively regulate the transcriptional activity of cell cycle genes. Cyclin-dependent kinase inhibitor $1 \mathrm{~B}$ (CDKN1B) is one of the effectors of E2F2, which is important in the cellular transition from quiescence to the proliferative state. HOXA1-stimulated oncogenicity is mediated by selective upregulation of components of the p44/42 MAP kinase pathway in human mammary carcinoma cells (32). The expression level of HOXA1 is correlated with poor prognosis of oral squamous cell carcinoma (33). The only TF of HOXA1 is wingless-type MMTV integration site family member $5 \mathrm{~A}$ (Wnt5a). It is reported that Wnt5a signaling is involved in the regulation of osteosarcoma cell invasiveness (34).

In conclusion, differential expression of several direct and indirect target genes of p53 was observed following treatment with Nutlin-3. These findings not only advanced the understanding regarding the importance of p53 in osteosarcoma, but also provided clues for future development of therapeutic strategies.

\section{References}

1. Espinosa JM: Mechanisms of regulatory diversity within the p53 transcriptional network. Oncogene 27: 4013-4023, 2008.

2. Menendez D, Inga A and Resnick MA: The expanding universe of p53 targets. Nat Rev Cancer 9: 724-737, 2009.

3. Riley T, Sontag E, Chen P and Levine A: Transcriptional control of human p53-regulated genes. Nat Rev Mol Cell Biol 9: 402-412, 2008.

4. Miller LD, Smeds J, George J, Vega VB, Vergara L, Ploner A, Pawitan Y, Hall P, Klaar S, Liu ET and Bergh J: An expression signature for $\mathrm{p} 53$ status in human breast cancer predicts mutation status, transcriptional effects and patient survival. Proc Natl Acad Sci USA 102: 13550-13555, 2005.

5. Wei CL, Wu Q, Vega VB, Chiu KP, Ng P, Zhang T, Shahab A, Yong HC, Fu Y, Weng Z, et al: A globalmap of 53 transcription-factor binding sites in the human genome. Cell 124: 207-219, 2006.

6. Bruna A, Darken RS, Rojo F, Ocaña A, Peñuelas S, Arias A, Paris R, Tortosa A, Mora J, Baselga J and Seoane J: High TGF $\beta$-Smad activity confers poor prognosis in glioma patients and promotes cell proliferation depending on the methylation of the PDGF-B gene. Cancer Cell 11: 147-160, 2007.

7. Rozan L and El-Deiry W: p53 downstream target genes and tumor suppression: A classical view in evolution. Cell Death Differ 14: 3-9, 2006

8. Botcheva K, McCorkle SR, McCombie W, Dunn JJ and Anderson CW: Distinct p53 genomic binding patterns in normal and cancer-derived human cells. Cell Cycle 10: 4237-4249, 2011.

9. Smeenk L, Van Heeringen SJ, Köppel M, Gilbert B Janssen-Megens E, Stunnenberg HG and Lohrum M: Role of p53 serine 46 in p53 target gene regulation. PloS One 6: e17574, 2011.
10. Smeenk L, van Heeringen SJ, Koeppel M, van Driel MA, Bartels SJ, Akkers RC, Denissov S, Stunnenberg HG and Lohrum M: Characterization of genome-wide p53-binding sites upon stress response. Nucleic Acids Res 36: 3639-3654, 2008.

11. Jaffe N, Bruland OS and Bielack S: Pediatric and adolescent osteosarcoma. Springer, New York, NY, pp275-287, 2010.

12. Ottaviani $\mathrm{G}$ and Jaffe N: The epidemiology of osteosarcoma. Cancer Treat Res, pp3-13, 2010.

13. Menendez D, Nguyen TA, Freudenberg JM, Mathew VJ, Anderson CW, Jothi R and Resnick MA: Diverse stresses dramatically alter genome-wide p53 binding and transactivation landscape in human cancer cells. Nucleic Acids Res 41: 7286-7301, 2013.

14. Gautier L, Cope L, Bolstad BM and Irizarry RA: Affy-analysis of Affymetrix GeneChip data at the probe level. Bioinformatics 20 : 307-315, 2004

15. Diboun I, Wernisch L, Orengo CA and Koltzenburg M: Microarray analysis after RNA amplification can detect pronounced differences in gene expression using limma. BMC Genomics 7: 252, 2006

16. Qin J, Li MJ, Wang P, Zhang MQ and Wang J: ChIP-Array: combinatory analysis of ChIP-seq/chip and microarray gene expression data to discover direct/indirect targets of a transcription factor. Nucleic Acids Res 39: W430-W436, 2011.

17. Huang DW, Sherman BT, Tan Q, Collins JR, Alvord WG, Roayaei J, Stephens R, Baseler MW, Lane HC and Lempicki RA: The DAVID Gene Functional Classification Tool: A novel biological module-centric algorithm to functionally analyze large gene lists. Genome Biol 8: R183, 2007.

18. Diller L, Kassel J, Nelson CE, Gryka MA, Litwak G, Gebhardt M, Bressac B, Ozturk M, Baker SJ and Vogelstein B: p53 functions as a cell cycle control protein in osteosarcomas. Mol Cell Biol 10: 5772-5781, 1990.

19. Overholtzer M, Rao PH, Favis R, Lu XY, Elowitz MB, Barany F, Ladanyi M, Gorlick R and Levine AJ: The presence of p53 mutations in human osteosarcomas correlates with high levels of genomic instability. Proc Natl Acad Sci USA 100: 11547-11552, 2003.

20. Berman SD, Calo E, Landman AS, Danielian PS, Miller ES, West JC, Fonhoue BD, Caron A, Bronson R, Bouxsein ML, et al: Metastatic osteosarcoma induced by inactivation of $\mathrm{Rb}$ and p53 in the osteoblast lineage. Proc Natl Acad Sci USA 105: 11851-11856, 2008

21. Nakase M, Inui M, Okumura K, Kamei T, Nakamura S and Tagawa T: p53 gene therapy of human osteosarcoma using a transferrin-modified cationic liposome. Mol Cancer Ther 4: 625-631, 2005.

22. Momand J, Wu H-H and Dasgupta G: MDM2-master regulator of the p53 tumor suppressor protein. Gene 242: 15-29, 2000.

23. Chène P: Inhibiting the p53-MDM2 interaction: An important target for cancer therapy. Nat Rev Cancer 3: 102-109, 2003.

24. Klein $\mathrm{C}$ and Vassilev LT: Targeting the p53-MDM2 interaction to treat cancer. Br J Cancer 91: 1415-1419, 2004.

25. Zhang Y, Shu L and Chen X: Syntaxin 6, a regulator of the protein trafficking machinery and a target of the $\mathrm{p} 53$ family, is required for cell adhesion and survival. J Biol Chem 283: 30689-30698, 2008.

26. Bouleau S, Grimal H, Rincheval V, Godefroy N, Mignotte B, Vayssière JL and Renaud F: FGF1 inhibits p53-dependent apoptosis and cell cycle arrest via an intracrine pathway. Oncogene 24: 7839-7849, 2005.

27. Sutton AL, Zhang X, Ellison TI and MacDonald PN: The 1, $25(\mathrm{OH})$ 2D3-regulated transcription factor MN1 stimulates vitamin D receptor-mediated transcription and inhibits osteoblastic cell proliferation. Mol Endocrinol 19: 2234-2244, 2005.

28. Zhang X, Dowd DR, Moore MC, Kranenburg TA, Meester-Smoor MA, Zwarthoff EC and MacDonald PN: Meningioma 1 is required for appropriate osteoblast proliferation, motility, differentiation and function. J Biol Chem 284: 18174-18183, 2009.

29. Zhu JW, Field SJ, Gore L, Thompson M, Yang H, Fujiwara Y, Cardiff RD, Greenberg M, Orkin SH and DeGregori J: E2F1 and E2F2 determine thresholds for antigen-induced T-cell proliferation and suppress tumorigenesis. Mol Cell Biol 21: 8547-8564, 2001.

30. Pusapati RV, Weaks RL, Rounbehler RJ, McArthur MJ and Johnson DG: E2F2 suppresses Myc-induced proliferation and tumorigenesis. Mol Carcinog 49: 152-156, 2010.

31. Laresgoiti U, Apraiz A, Olea M, Mitxelena J, Osinalde N, Rodriguez JA, Fullaondo A and Zubiaga AM: E2F2 and CREB cooperatively regulate transcriptional activity of cell cycle genes. Nucleic Acids Res 41: 10185-10198, 2013. 
32. Mohankumar KM, Xu X, Zhu T, Kannan N, Miller LD, Liu ET, Gluckman PD, Sukumar S, Emerald BS and Lobie PE: HOXA1-stimulated oncogenicity is mediated by selective upregulation of components of the p44/42 MAP kinase pathway in human mammary carcinoma cells. Oncogene 26: 3998-4008, 2007.

33. Bitu CC, Destro MF, Carrera M, da Silva SD, Graner E, Kowalski LP, Soares FA and Coletta RD: HOXA1 is overexpressed in oral squamous cell carcinomas and its expression is correlated with poor prognosis. BMC cancer 12: 146, 2012.
34. Enomoto M, Hayakawa S, Itsukushima S, Ren DY, Matsuo M, Tamada K, Oneyama C, Okada M, Takumi T, Nishita M, et al: Autonomous regulation of osteosarcoma cell invasiveness by Wnt5a/Ror2 signaling. Oncogene 28: 3197-3208, 2009. 